\title{
A CHARACTERIZATION OF ENTIRE FUNCTIONS IN TERMS OF DEGREE OF CONVERGENCE
}

\author{
BY JOHN R. RICE
}

Communicated by Eugene Isaacson, July 10, 1969

1. The result. Consider a point set $C$ in the complex plane whose complement $K$ is connected and regular (i.e. $K$ possesses a Green's function with pole at infinity). Let $d_{\infty}$ denote the transfinite diameter of $C$. Recall that $d_{\infty}=1 /\left|\phi^{\prime}(\infty)\right|$ where $\phi(z)$ maps $K$ onto the exterior of the unit circle. Equivalently, $d_{\infty}=\operatorname{Lim}_{n \rightarrow \infty}\left[\left\|T_{n}(z)\right\|_{c}\right]^{1 / n}$ where $\|g(z)\|_{C}=\max |g(z)|, z \in C$ and $T_{n}(z)$ is the Tchebycheff polynomial (or Faber polynomial) for $C$. Given $f(z)$ defined on $C$, let $P_{n}^{*}(z)$ be the best polynomial approximation to $f(z)$ on $C$ i.e., $\left\|f(z)-P_{n}(z)\right\|_{C}$ is minimized for polynomials $P_{n}(z)$ of degree $n$. The purpose of this note is to announce the

Theorem. We have

$$
\operatorname{Lim}_{n \rightarrow \infty} n^{1 / \rho}\left\|f(z)-P_{n}^{*},(z)\right\|^{1 / n}=d_{\infty}(e \rho \tau)^{1 / \rho}
$$

if and only if $f(z)$ is entire of order $\rho>0$ and type $0<\tau<\infty$.

The proof of this is to appear elsewhere. The method of proof essentially combines basic techniques from the theory of entire functions with machinery used to establish degree of convergence theorems for polynomial approximations to analytic functions. Thus it is shown that this degree of convergence is achieved (with a factor $1+\epsilon$, arbitrary $\epsilon>0$ ) by a polynomial expansion of the form

$$
f(z)=\sum_{k=1}^{\infty} q_{k}(z) p(z)^{k-1}
$$

where $p(z)$ is a polynomial of degree $\lambda$ and $q_{k}(z)$ is of degree $\lambda-1$. The level curves of $|p(z)|$ define a lemniscate which approximates the boundary of $C$. A number of lemmas are established which relate the nature of the coefficient polynomials $q_{k}(z)$ to the order and type of $f(z)$.

AMS Subject Classifications. Primary 4141, 3055; Secondary 3057, 4117, 4115. Key Words and Phrases. Degree of convergence, entire functions, transfinite diameter, polynomial approximation.

Purdue University, West Lafayette, Indiana 47907 